\title{
THE USE OF ULTRASONOGRAPHY IN DIAGNOSTIC IMAGING OF REPTILES
}

\author{
Urbanová, D., Halán, M. \\ Institute of Parasitology \\ University of Veterinary Medicine and Pharmacy, Komenského73, 04181 Košice \\ The Slovak Republic \\ milos.halan@uvlf.sk
}

\section{ABSTRACT}

The aim of this study was to obtain new knowledge and show the possibility of the use of ultrasonographic (USG) examinations in reptilian medicine. As reptiles are patients brought to veterinary clinic in smaller numbers, we focused on the indications and limits of this type of examination in individual groups of reptiles. In the period of 2014-2015 we examined by ultrasound, 28 reptiles with the aim to diagnose gravidity, reproductive problems and to determine their gender. At the same time we examined the internal organs and their availability for potential USG examinations. We also investigated: the issues related to fixation and positioning of the patients; selection of suitable examination probes; and the ways of their application to a suitable body area. The experimental part of our study was focused on the examination of the reproductive apparatus of reptiles. We monitored: individual phases of gravidity in selected reptilian species; evaluated development of follicles in ovaries; and the development of eggs after successful mating up to their laying. We described the pathological states of reproductive organs and the suitability of this examination for the determination of gender.

Key words: diagnostic imaging; lizard; reptile; snake; tortoise; turtle; ultrasonography

\section{INTRODUCTION}

Ultrasonography (USG) is an important diagnostic tool in any veterinary practice with many potential uses in reptilian medicine. In general, this examination is considered safe for both the patient and the examination personnel. It is a non-invasive method for the evaluation of anatomical size, position and structure of the internal organs. Neither the patient nor the assisting personnel are exposed to ionizing radiation [5].

The organs best suitable for examination by USG include: the heart, liver, gallbladder, urinary bladder, large intestine and gonads. In some animals, USG enables the examination of: the stomach, small intestine, spleen, pancreas and kidneys [6]. 
The examination by ultrasound allows one to evaluate: inflammatory changes, tumours, developmental irregularities, presence of foreign bodies, degeneration of tissues, and to confirm gravidity and carry out other investigations [3].

USG is a suitable method for the observation of reproductive functionality, reproductive cycle and diseases of reproductive organs. Other indications include the determination of gender, evaluation of reproductive potential or assessment of therapeutic intervention. The determination of gender on the basis of external morphology is frequently quite demanding. Ultrasonographic examination appears to be a useful method for the confirmation of gender in reptilian species [4].

With regard to the age of the patient and stage of the reproductive cycle, reptilian testicles are ovoid structures of varying size from hardly visible up to distinctive. They are especially prominent in Green iguana and Bearded dragon during the reproduction season. They have distinct granular homogenous echotexture, are isoechogenic, even somewhat more hyperechogenic than the liver [2].

The ovaries are visible depending on their size and phase of the reproductive cycle. At the beginning of this cycle the ovarian follicles are anechogenic. Their size varies from $1 \mathrm{~mm}$ up to several $\mathrm{mm}$. Before ovulation, they grow, appear as large circular structures and their echogenicity increases. In the late pre-ovulation phase almost the entire abdominal cavity is filled up with round follicles. After ovulation, the eggs grow and the wall turns hyperechogenic. Depending on the species, the wall may calcify or not at all. Monitoring of the inside of the egg may be based on the degree of calcification. At the beginning of gravidity, we can distinguish in the internal content, the anechogenic egg white layer and hyperechogenic yolk. Sometimes a very little embryo can also be visible. In viviparous reptiles one can observe the development of the embryo, developmental phase, embryonal activity and the heartbeat [2].

The aim of this study was to obtain new knowledge and show new possibilities of the use of (USG) examination in reptilian medicine with a focus on the reproductive cycle in selected reptilian species.

\section{MATERIALS AND METHODS}

Examinations were carried out employing a USG instrument Aloka prosound. We used either linear or convex probe. The linear probe of frequency $10-15 \mathrm{MHz}$ was preferred for examination of snakes and lizards, and tortoises and turtles were examined by means of a convex probe of frequency $3.5-7 \mathrm{MHz}$.

\section{The following reptiles and organs were examined by USG:}

- Tortoises and turtles $(\mathrm{n}=7)$ :

Hermann's tortoise (Testudo hermani): 1 male heart,

Pond slider (Trachemys scripta scripta): 3 females heart, liver, reproductive apparatus,

Red-eared slider (Trachemys scripta elegans): 3 females - reproductive apparatus.

- Lizards $(\mathrm{n}=16)$ :

Bearded dragon (Pogona vitticeps): 1 male +4 females - heart, liver reproductive organs,

Leopard gecko (Eublepharis macularius): 5 females reproductive apparatus,

Gila monster (Heloderma suspectum): 1 male +1 female - liver, heart, gallbladder, reproductive organs,

Veiled chameleon (Chameleon calyptratus): 4 females - reproductive organs.

- Snakes $(\mathrm{n}=5)$ :

Indochinese spitting cobra (Naja siamensis): 1 female - reproductive apparatus,

Ocellated carpet viper (Echis ocellatus): 1 female reproductive apparatus,

Prairie rattlesnake (Crotalus viridis): 1 male +1 female - hemipenises in male; reproductive apparatus in female,

Ornate flying snake (Chrysopelea ornata): 1 female reproductive apparatus, liver.

\section{RESULTS}

Our study included observations of the course of gravidity in four Leopard gecko females (Eublepharis macularius). Examinations of females were carried out in regular intervals. First we observed the presence of round follicles and after fertilization, the formation and growth of eggs until laying.

The first examination on March 11th showed the presence of round follicles about $5 \mathrm{~mm}$ in diameter. By April 1st the follicles reached a mean size of $8.6 \times 9.2 \mathrm{~mm}$. 


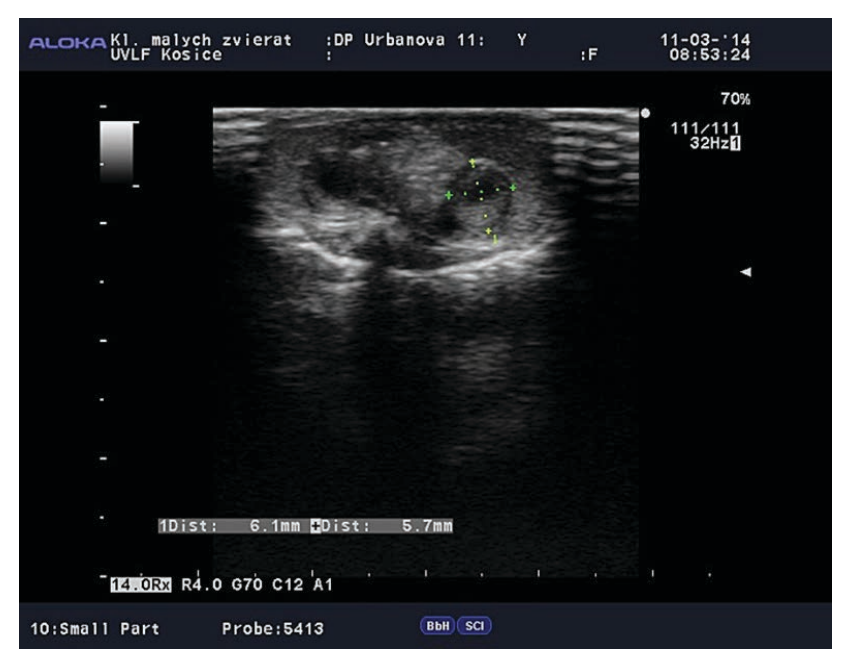

Fig. 1. First examination on March 11th

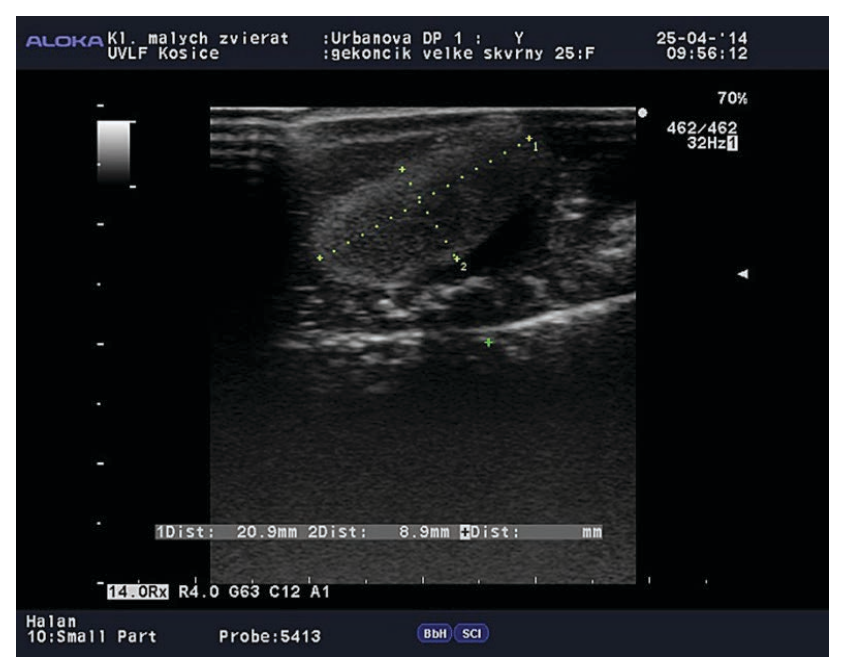

Fig. 3. Examination on April 25th

Table 1. Mean size of follicles and eggs of Leopard geckoes at individual examinations

\begin{tabular}{cc} 
Date & $\begin{array}{c}\text { Mean size } \\
{[\mathrm{mm}]}\end{array}$ \\
\hline March 11 & $5 \times 5$ \\
April 1 & $8.6 \times 9.2$ \\
April 25 & $9.3 \times 18$ \\
May 15 & $13.7 \times 28.4$ \\
\hline
\end{tabular}

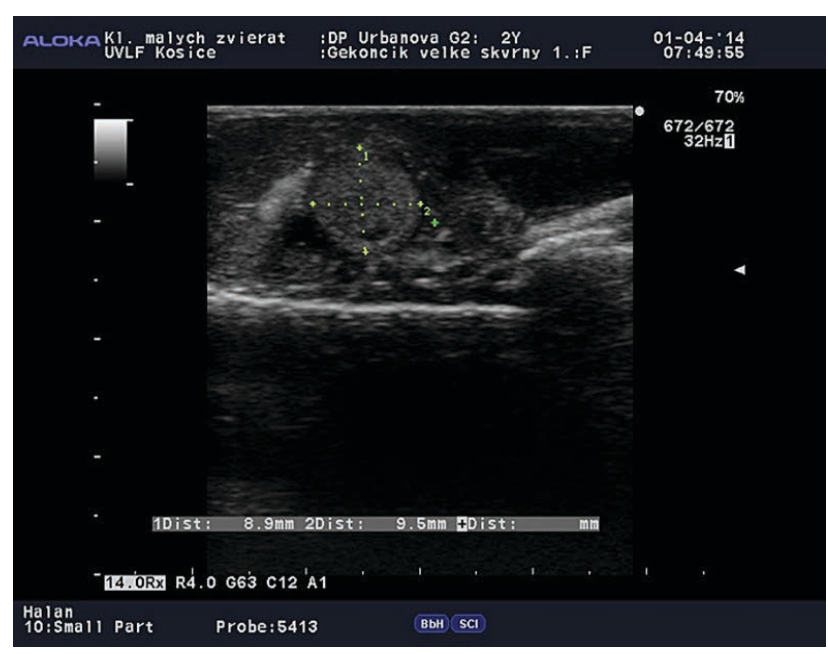

Fig. 2. Examination on April 1st

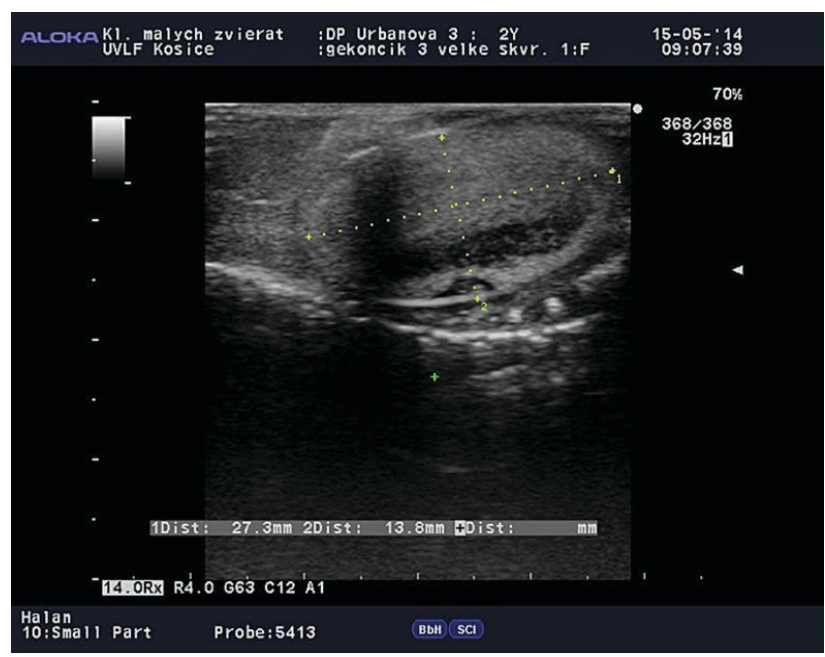

Fig. 4. Examination on May 15th

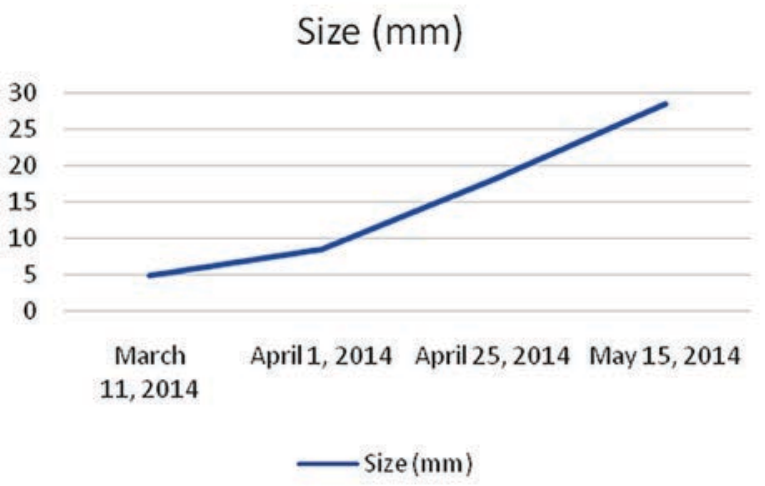

Fig. 5. Development of follicles and eggs in Leopard gecko females 


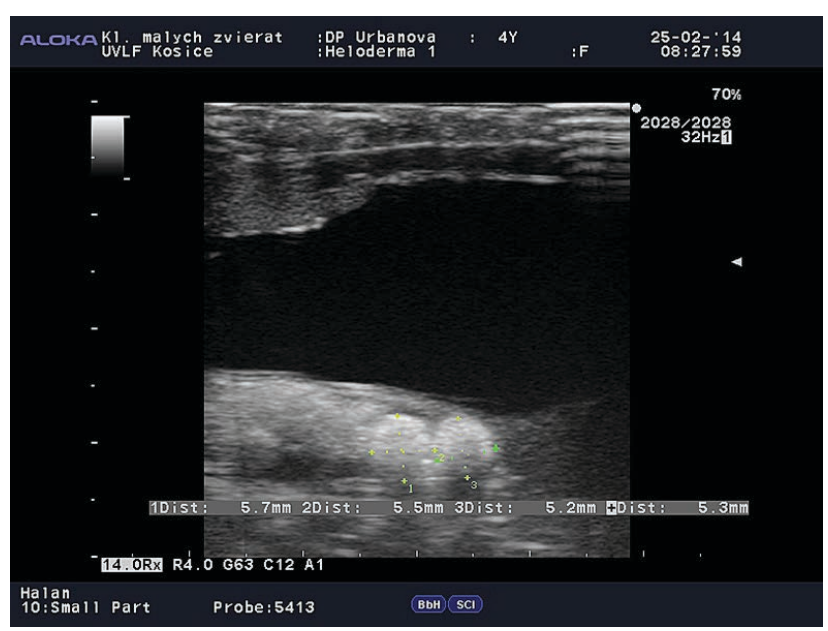

Fig. 6. Examination of Gila monster female on February 25

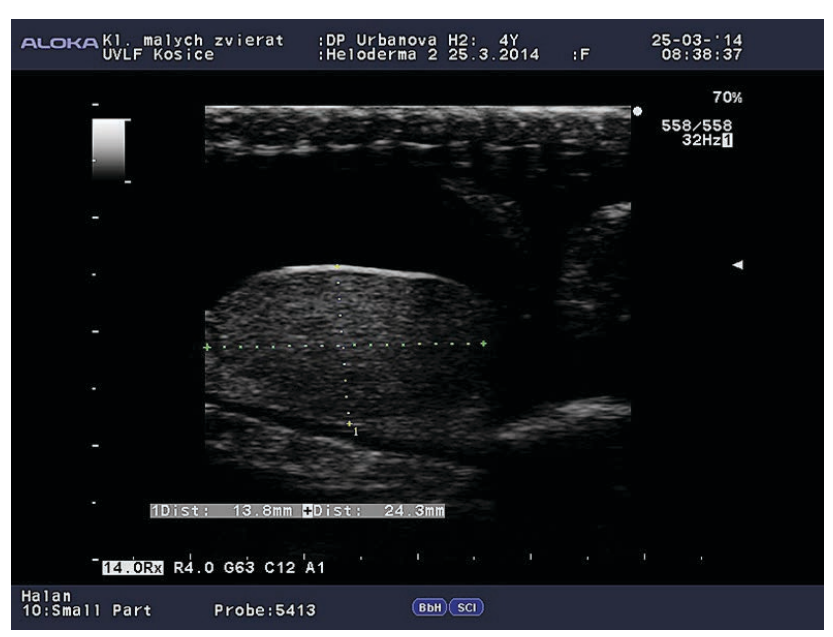

Fig. 7. Examination of Gila monster on March 25

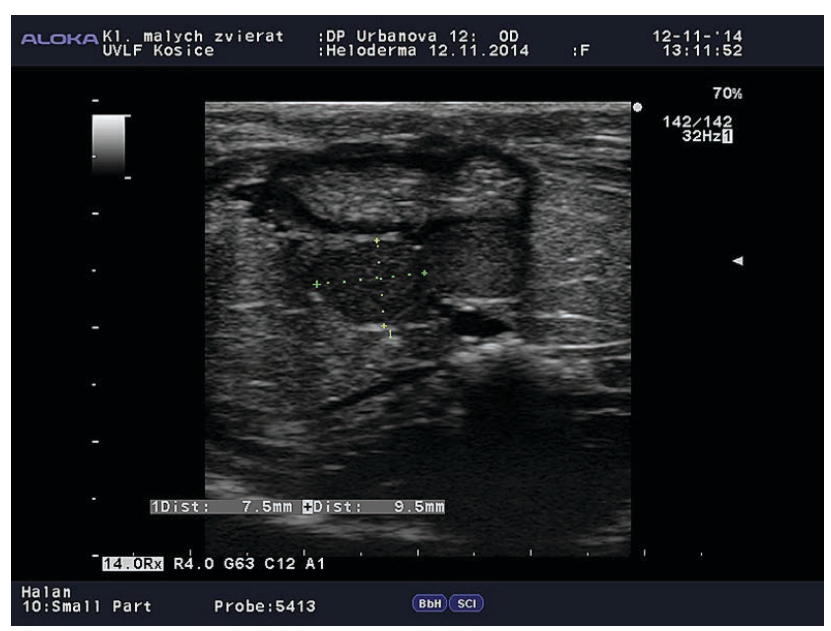

Fig. 8. Examination of Gila monster on November 12

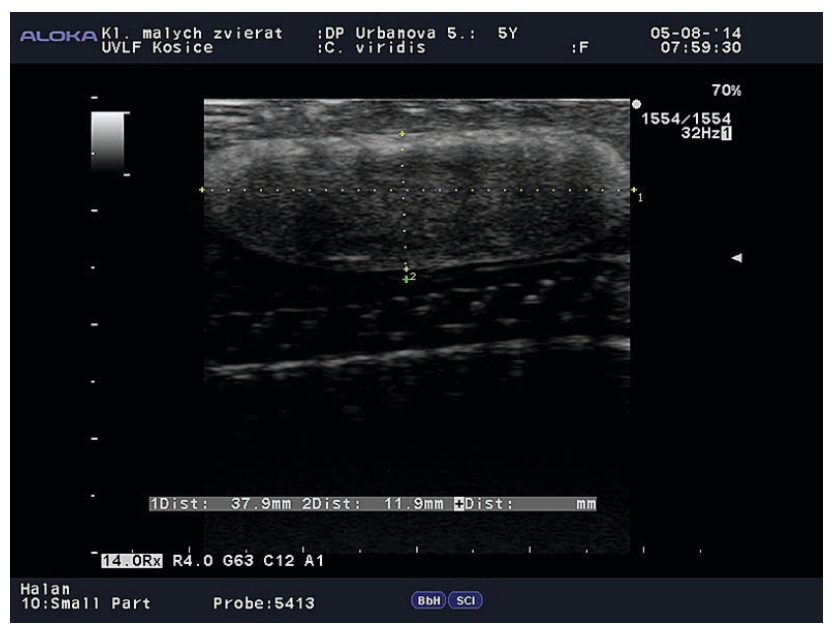

Fig. 9. Examination of Prairie rattlesnake female on August 5

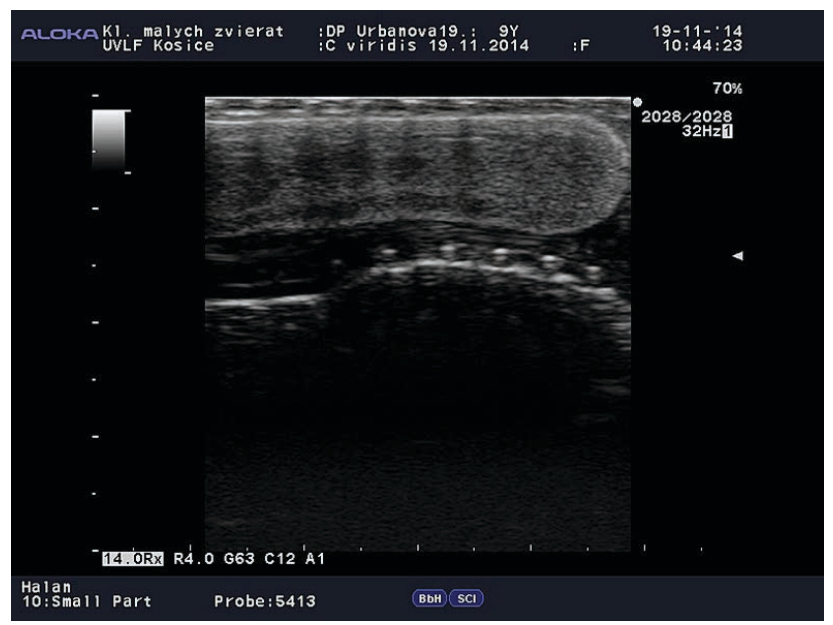

Fig. 10. Examination on August 26 


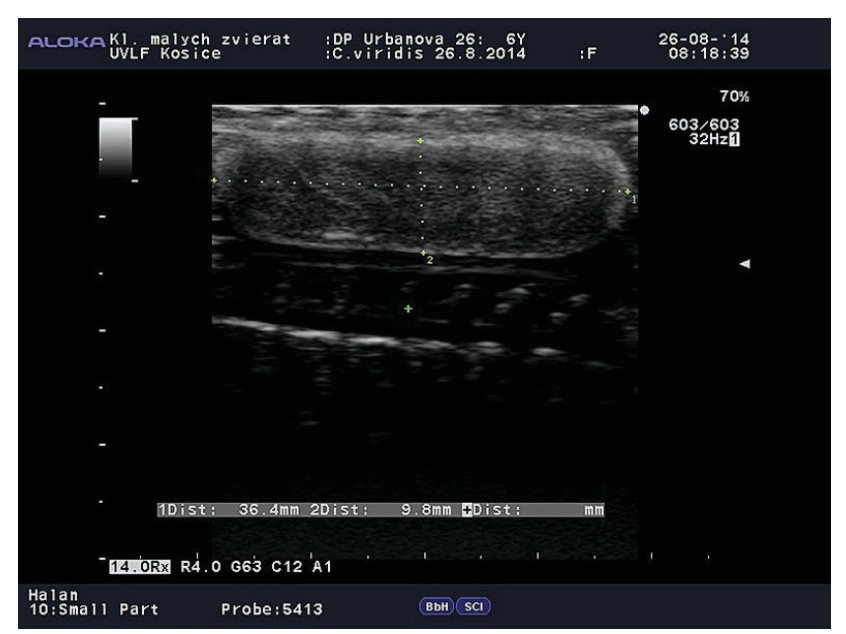

Fig. 11. Examination on September 23

The following examination on April 25th showed the presence of oval-shaped eggs covered in a hyperechogenic surface envelope which filled up most of abdominal cavity. Their mean size was $9.3 \times 18 \mathrm{~mm}$. By May $15 \mathrm{th}$, the eggs grew to the mean size of $13.7 \times 28.4 \mathrm{~mm}$. The eggs of this size could be observed by a naked eye through abdominal wall. Three days later the eggs were laid.

We also observed the reproductive cycle of a young Gila monster (Heloderma suspectum) female at first mating which resulted in unsuccessful gravidity. The first examination of this female on February 25th showed the presence of round follicles with a diameter of $5.5 \mathrm{~mm}$. One month later, oval-shaped eggs started to develop and reached the size of $13.8 \times 24.3 \mathrm{~mm}$. Afterword, their development evidently stopped and the eggs were absorbed. The examination on November 12 showed again the presence of round follicles $8.5 \mathrm{~mm}$ in diameter.

We examined one viviparous Prairie rattlesnake (Crotalus viridis) female for the presence and growth of eggs and the gradual development of embryos. During the first examination on August 5th, we observed eggs of homogeneous structure of size $37.9 \times 11.9 \mathrm{~mm}$. Three weeks later the egg size was $36.4 \times 9.8 \mathrm{~mm}$ and there were no signs of the development of the foetuses. At the following examination (September 23rd), there were still present eggs of homogenous structure of size $33 \times 9.6 \mathrm{~mm}$, but the developing foetuses were absent which indicated abnormal gravidity. Large homogeneous eggs were still present on November 19 th, confirming unsuccessful fertilization. Several days later, the unfertile eggs were disposed of by the female.

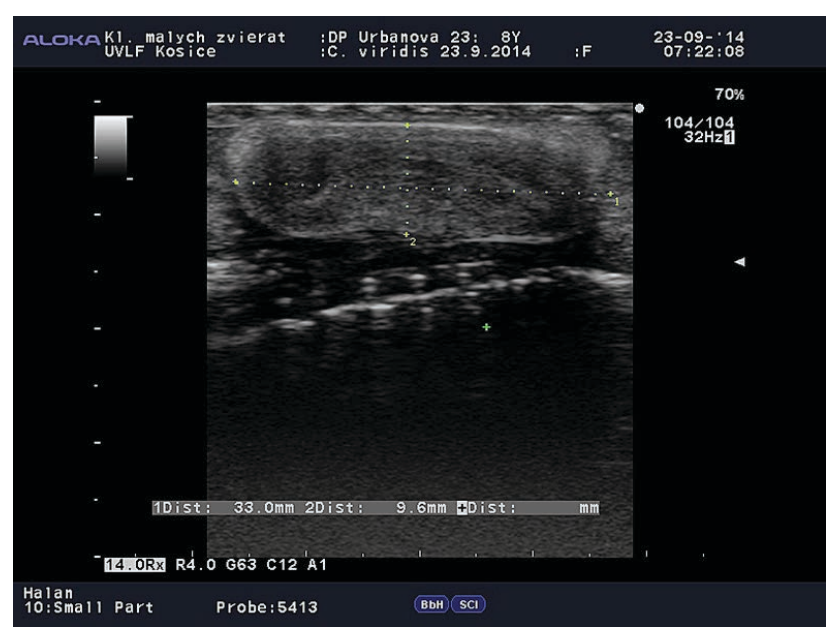

Fig. 12. Examination on November 19

In addition to the examination of the reproductive apparatus of the females, we examined in individual reptile species also, heart, liver, gallbladder and reproductive organs of males.

During ultrasonographic examination the heart served as a starting point which allowed us to orientate oneself to the internal structures. The heart of reptiles is three chambered, composed of two atria and one ventricle. During examination, one can visualize the entire cardiac cycle. Systole represents the time during which the ventricle contracts and ejects blood into the circulation. Diastole represents the period of time when all heart cavities are filled up gradually. The flow of blood can be observed by Doppler imaging.

The liver of reptiles appears ultrasonographically as homogenous hyperechogenic tissue. It is located largely in the cranial part of abdominal cavity. During examination we were able to visualise anechogenic vessels the presence of which can be confirmed by Doppler. In the tortoises, the cervicobracheal region is used for the examination of the liver.

The gallbladder of reptiles is localised in the abdominal cavity caudally of the liver, and in snakes, at relatively a long distance from the caudal tip of the liver. In snakes the gallbladder was not well developed. During the examination of the gallbladder, we were able to observe a thin hyperechogenic wall with anechogenic content. Of pathological states, it is possible to discern urinary stones and thickened bladder wall due to irritation or inflammatory processes.

In male reptiles one can observe the testes in the sea- 
sonal reproductive cycle. One of the characteristic features of snakes and lizards are hemipenises, the paired structures found behind the cloaca. For their imaging, the probe should be applied behind the cloaca and gradually moved along to the ventral basis of the tail. They appear as heterogenous structures surrounded by muscles.

\section{DISCUSSION}

Recently, the interest in rearing terrarium animals has increased. Thus, veterinarians face more frequently issues related to reptilian reproduction. Ultrasonography appears to be a useful diagnostic tool also in these animals.

Girling and Raiti described many indications for which USG examination can be considered as useful. They include: determination of gender and the stage of the reproductive cycle, confirmation of gravidity, examination of soft tissues, intraocular structures, vascularization of tissue, and detection of pathological changes and tumours [1].

However, there are some limits which can complicate this examination. The problems may be related to very small size of some individuals, the period of shedding, manipulation, fixation and the way of application of the probe [6].

In our study we used USG imaging to examine: the heart, liver gallbladder and reproductive organs in males and females of different reptile species. One part of our study focused on the examination of the reproductive apparatus of female reptiles.

Successful imaging of ovaries is dependent on their size and the reproductive phase. In the period of sexual activity they are discernible on the basis of the presence of round follicles. At the onset of gravidity, oval-shaped eggs are formed in the oviduct and their surface gradually calcifies which is manifested by a surface hyperechogenic layer. In viviparous reptiles, one can observe the development and the activity of the foetuses [5].

Our observations of the reproductive apparatus of Red eared slider (Trachemis scripta elegans) indicated preovulatory follicle stasis (POFS) which was detected also in Veiled chameleon (Chameleon calyptratus).

The examination of the Leopard gecko (Eublepharis macularius) females allowed us to observe the presence of round follicles and after mating, the formation and growth of eggs up to their laying. The mean size of eggs increased from $18 \times 9.3 \mathrm{~mm}$ up to $28.4 \times 13.7 \mathrm{~mm} 3$ days before laying when it was possible to observe them with the naked eye through the abdominal wall.

The reproductive cycle of a young Gila monster (Heloderma suspectum) female at first mating observed during our study was unsuccessful, although its eggs started to develop and reached a size of $13.8 \times 24.3 \mathrm{~mm}$. However, they were subsequently absorbed and round follicles appeared again.

In one viviparous Prairie rattlesnake (Crotalus viridis) we observed unfertilised eggs which were retained for almost four months, but decreased in size and finally were disposed of by the female.

The determination of gender in species with minimum sexual dimorphism can present a problem for both veterinarians and breeders and USG examination appears to be suitable for this purpose. In males one can observe the testes or hemipenises, and in females, round follicles in ovaries or oval eggs in the oviducts. Our examination of two male reptiles, one Gila monster lizard (H. suspectum) and one Prairie rattlesnake (C. viridis) confirmed the presence of hemipenises in these reptiles. It is a paired organ visible at the tail basis behind the cloaca. By this examination, one can identify males without manual probing and thus eliminate the risk of trauma to hemipenises or cloaca in these animals [7].

\section{CONCLUSIONS}

Our study investigating the possibility of the examination of individual internal organs in selected reptile species showed that USG imaging is most suitable for the examination of the liver, heart, gallbladder and reproductive organs. By this method, it is possible to evaluate some physiological parameters, such as size, position, echogenicity, blood flow by Doppler imaging and also to detect some pathological deviations.

In selected females of lizards and snakes, it was possible to observe the phases and course of gravidity, development and expulsion of unfertilized eggs by viviparous female of Prairie rattlesnake and unsuccessful gravidity in a young Gila monster lizard. This examination is non-invasive and may replace some other potentially risky approaches used for determination of gender.

On the basis of the above, one may conclude that USG imaging is a diagnostic tool suitable also for exotic animals 
such as reptiles. However, it is necessary to observe all technical requirements and consider the anatomic-morphological specificities of individual reptile species.

\section{REFERENCES}

1. Girling, S. J., Raiti, P., 2004: Manual of Reptiles. 2nd edn., BSAVA, 392 pp.

2. Mader, D. R., Divers, J.S., 2013: Current Therapy in Reptile Medicine and Surgery. Saunders, $488 \mathrm{pp}$.

3. Nylannd, T. G., Matton J. S., 2002: Small Animal Diagnostic Ultrasound. 2nd edn., Saunders, $461 \mathrm{pp}$.

4. Pees, M., Hänse, M., Schlömer. J., Schroff, S., 2010: Legenot bei Reptilien. Ausgewählter Kongressbeitrag, 38, 69-72.
5. Schumacher, J., Toal, R.L., 2001: Advanced radiography and ultrasonography in reptiles. Seminars in Avian and Exotic Pet Medicine, 10, 162-168.

6. Stetter, M., 2000: Diagnostic imaging of reptiles. In Kirk R. (Ed.): Current Veterinary Therapy XIII: Small Animal Practice, WB Saunders, Orlando, Florida, 1163-1168.

7. Pees, M., Hänse, M., Schlömer, J. et al., 2010: Legenot bei Reptilien. Tierärztl. Prax, 38, 69-72.

Selected paper from the 59th STUDENT SCIENTIFIC CONFERENCE, Section II - Clinical section, held at the University of Veterinary Medicine and Pharmacy in Košice, SR, on April 6, 2016. 